\title{
Above and beyond: an exploratory study of breast cancer patient accounts of healthcare provider information-giving practices and informational support
}

\author{
Andrea L. Meluch \\ Department of Communication Studies, Indiana University South Bend, South Bend, Indiana, USA
}

\begin{abstract}
This qualitative study examines breast cancer patients' accounts of the characteristics of healthcare providers' supportive information-giving practices. Twenty-two women diagnosed with breast cancer participated in semi-structured in-depth interviews designed to understand their experiences receiving supportive information from healthcare providers (e.g., oncologists, surgeons, nurse practitioners). Participants' accounts suggest that providers who spend extensive time discussing cancer-related information and who explain that information thoroughly so that patients can understand their medical situation, are communicating informational support in contrast to merely presenting factual information related to cancer diagnosis and treatments. Participant accounts further suggest that the supportive nature of provider information-giving practices results from message framing, or the provider's metacommunication.
\end{abstract}

\section{Introduction}

Breast cancer is the most common type of cancer and a leading cause of death among women in the U.S. ${ }^{1}$ Breast cancer diagnoses vary widely and patients diagnosed with breast cancer require treatment regimens personalized for individual needs. ${ }^{2}$ As such, women diagnosed with breast cancer have a need for both quality cancer-related information $^{3}$ and social support. ${ }^{4-8}$ Healthcare professionals, such as physicians and nurses, often are a primary source of cancer-related information ${ }^{3}$ and social support ${ }^{6}$ for women di-

Correspondence: Andrea L. Meluch, Department of Communication Studies, Indiana University South Bend, 1700 Mishawaka Avenue, South Bend, Indiana 46615, USA.

Tel.: 574.520.5232.

E-mail: ameluch@iusb.edu

Key words: Informational support; Breast cancer; Information-giving; Healthcare; Metacommunication.

Conflict of interest: the author declares no potential conflict of interest.

Funding: none.

Received for publication: 28 February 2018.

Revision received: 21 July 2018.

Accepted for publication: 21 July 2018.

This work is licensed under a Creative Commons Attribution NonCommercial 4.0 License (CC BY-NC 4.0).

(C) Copyright A.L. Meluch, 2018

Licensee PAGEPress, Italy

Qualitative Research in Medicine \& Healthcare 2018; 2:113-120

doi:10.4081/qrmh.2018.7387 agnosed with breast cancer. Research has long indicated that people diagnosed with cancer have increased support needs ${ }^{9}$ and that healthcare providers often help to meet these support needs. ${ }^{6,89}$ Studies examining the support provided by healthcare professionals have focused on the source of the support (e.g., physician, nurse), the function of the support provided (e.g., informational, emotional), and the health outcomes related to patients' use of support. ${ }^{6}$

Cancer is a complex diagnosis and, as such, people diagnosed with cancer receive complex information and experience a greater number of interactions with healthcare providers. ${ }^{3}$ Many research studies have cast cancer-related information provided to patients by providers as a type of social support. ${ }^{4,5,8}$ However, limited research has investigated how the manner used to provide cancer-related information leads to patients feeling supported rather than overwhelmed and unsatisfied. ${ }^{6}$ That is, much of the research on the informational support provided to those diagnosed with cancer classifies the cancer-related information delivered to patients by healthcare providers as supportive, but does not distinguish whether patients feel the information they received has met their needs as opposed to actually considering it delivered in a supportive manner. Research suggests that healthcare providers do not always provide adequate amounts of cancer-related information to patients and patients may be left with unmet needs. ${ }^{10}$ Further, when patients' information needs are not fully met, they may have worsened health outcomes as a result. ${ }^{3}$ However, patients report more satisfaction with their medical encounter and experience various health benefits when they receive informational support. ${ }^{6,11}$ Thus, understanding the characteristics of information delivery patients report as supportive may assist researchers in ensuring that patient information needs are met. The present study examines the ways in which 
healthcare professionals convey information in a supportive manner to breast cancer patients.

\section{Social support}

Social support is a communicative process that results in the construction of social networks. ${ }^{12}$ Albrecht and Adelman ${ }^{13}$ defined social support as verbal and nonverbal communication between recipients and providers that helps reduce uncertainty about the situation, the self, the other or the relationship and functions to enhance a perception of personal control in one's life experience. Albrecht and Adelman's definition argued that social support reduces uncertainty, or the ambiguity, that one experiences when one does not have complete information. ${ }^{14}$ For example, one encounters many unknowns when diagnosed with breast cancer (e.g., duration of illness, outcome of illness) and these unknowns can create feelings of uncertainty about numerous aspects of the situation. Albrecht and Adelman ${ }^{13}$ explained that uncertainty in health crises leads to stress. They contended that when social support reduces uncertainty, social support also reduces stress. Thus, Albrecht and Adelman initially argued that social support benefited health by reducing stress.

Albrecht and Goldsmith ${ }^{12}$ later revised their ${ }^{13}$ definition of social support to argue that social support helps individuals manage, instead of reduce, uncertainty. For example, people diagnosed with cancer can manage uncertainty related to treatment decisions when they receive informational support, thereby, improving their decision making and problem solving. ${ }^{6,15}$ Albrecht and Goldsmith ${ }^{12}$ also recognized that uncertainty is not necessarily a negative experience. Although an individual often experiences uncertainty negatively (e.g., concerns over one's ability to pay medical expenses or one's ability to manage side effects of chemotherapy treatment), uncertainty also can be a positive experience if it allows an individual to feel hopeful or optimistic. For example, people diagnosed with advanced cancer often want their healthcare providers to communicate some level of ambiguity about the future so that they can retain a sense of hope and even optimism regarding length and quality of life. ${ }^{16}$ Thus, social support helps individuals better manage the uncertainty accompanying their cancer diagnosis and experience reduced stress as a result.

The functions served by social support have interested researchers for several decades. ${ }^{12}$ Early on, $\mathrm{Cobb}^{17} \mathrm{ex}-$ plained that social support can provide resources (e.g., information, material aid, encouragement) that help individuals moderate their stress. Researchers later developed typologies of the functions that social support serves. ${ }^{18,19}$ Today, researchers often focus on three functions of social support (informational, emotional, instrumental).$^{20}$ Researchers interested in the social support people diagnosed with cancer receive from their healthcare providers commonly examine the informational function of social support. ${ }^{6}$

\section{Informational support}

Information, explanations, guidance, and advice can be understood as informational support. ${ }^{20}$ Sources of informational support for people diagnosed with cancer include family and friends, ${ }^{4}$ peers patients interact with through digital forums, ${ }^{21}$ and healthcare providers. ${ }^{4,6} \mathrm{Can}-$ cer patients often indicate an increased desire to receive informational support from their healthcare providers. ${ }^{5,6,8}$ Most patients receive substantial information from their healthcare providers and providing information in itself is a responsibility associated with healthcare providers' jobs or organizational roles. ${ }^{3,22}$ That is, healthcare providers have the responsibility to provide patients with the information they need to make informed decisions about their treatment. ${ }^{3,15,22}$

For patients, informational support can be a critical resource in helping one manage uncertainty, feel a greater sense of control over the situation, and experience health benefits. ${ }^{5,6,11}$ Mills and Sullivan ${ }^{11}$ reviewed literature regarding relationships between informational support offered by nurses to people diagnosed with cancer and those patients' health outcomes. They found associations between the informational support provided by nurses and reduced anxiety, enhanced sense of personal control, greater compliance with suggested medical regimens, and feelings of greater security. However, cancer patients do not always see information-giving processes as helpful because the amount or complexity of information can be overwhelming and contribute to greater stress and uncertainty. ${ }^{16,23}$ Thus, receiving information from a healthcare provider in of itself is not necessarily a supportive experience leading to health benefits.

Numerous research studies have indicated that people diagnosed with cancer classify the information that they receive from their healthcare providers as informational support. ${ }^{4,5,8,15}$ For example, Nazione et al. ${ }^{5}$ examined the verbal informational support surgeons provide to breast cancer patients. They operationalized informational support as any suggestion or advice...evaluation of the problem... [and] form of teaching the patient about the problem in an effort to problem solve. ${ }^{5}$ Nazione et al.'s operationalization of social support focuses on the content of the message, or verbal communication of information, and the outcomes of that information (i.e., making decisions about treatment). However, this operationalization of information-giving does not fully explain what it is about the way providers present information to patients that indicates the supportive nature of the communication (i.e., helping them manage uncertainty) in contrast to it being overwhelming, confusing, and/or unhelpful. Given that the majority of researchers have focused mainly on the informational content provided to patients (e.g., as advice, as a description intended to teach the patient) instead of whether other characteristics of provider information-giving (e.g., amount of information given) are indicative of supportive communication, the present exploratory study 
seeks to fill this gap. Thus, the following research question guides this study: What are the characteristics of healthcare provider information-giving processes that breast cancer patients report as indicating support?

\section{Materials and Methods}

The data used in this study are a subset of a larger existing dataset gathered to investigate cancer patient experiences of social support in healthcare settings. I conducted semi-structured in-depth interviews with individuals diagnosed with various types of cancer and focused on the interviews with women diagnosed with breast cancer in the present analysis.

\section{Participant demographics}

Participants' ages ranged from 34-82 years-old $(M=59.59, S D=10.73)$. Twenty participants self-identified as white and two participants self-identified as white and Native American. Participants were at different stages in their breast cancer treatment. The majority of participants (14) were married, four participants were divorced, two participants were widowed, and two participants had never been married. Three participants did not have children, four participants had one child, ten participants had two children, three participants had three children, one participant had four children, and one participant had six children. Five participants had obtained a high school diploma or GED, three participants had attended some college, six participants had obtained an associate's degree, five participants had obtained a bachelor's degree, and three participants had earned a graduate degree.

\section{Recruitment and data collection procedures}

I recruited participants at a cancer wellness center located in a medium-sized Midwestern city. Before the interviews took place, I conducted an informed consent process with all interviewees. The university Institutional Review Board approved the interview protocol used. I audio-recorded all interviews.

I conducted in-depth interviews with all study participants. In-depth interviews are the use of interviewing techniques to gather information and knowledge-usually deeper information and knowledge than is sought in surveys, informal interviewing, and focus groups. ${ }^{24}$ Using Charmaz's ${ }^{25}$ intensive interviewing process, I engaged in a one-sided conversation in which participants shared with me their personal experiences. Intensive interviews focus on a particular topic of which the participant has first-hand experience..$^{25}$ For example, in the present study participants shared their first-hand experiences interacting with healthcare providers throughout their breast cancer treatment.

To assist in focusing the conversation on the topic of interest in the present study, I followed an interview proto- col that focused on patients' experiences of support and nonsupport in their personal network (e.g., family friends), at a cancer wellness center, and with healthcare providers. Specifically, the interview protocol included questions asking participants about: i) instances of support and nonsupport from family, friends, and colleagues; ii) experiences interacting with other people diagnosed with cancer and participating in activities at the cancer wellness center; iii) their healthcare experiences related to cancer; iv) the extent to which participants felt supported by healthcare providers (e.g., physicians, nurses) and specific instances or examples of support (or nonsupport) during their cancer care; and vi) their accounts of the role of healthcare organizations and healthcare providers in society. Interviews ranged in length from 40 minutes to 118 minutes $(M=60.86$ minutes; $S D=15.79)$. Transcripts were encrypted and sent to Rev.com for transcription. After I received completed transcripts I de-identified them and assigned pseudonyms to each participant. Pseudonyms are used throughout the findings to refer to participants.

\section{Data analysis}

I implemented a constructivist grounded theory approach in the analysis of transcripts Constructivist grounded theory is an interpretive, systematic, and flexible method of qualitative analysis using comparative methods. ${ }^{25}$ A constructivist grounded theory approach to data analysis acknowledges the socially constructed nature of reality and the researchers' construction and interpretation of the qualitative data. ${ }^{25}$ Specifically, this approach acknowledges the researcher's role in creating the context in which the research is conducted and the process through which it is interpreted. As such, the findings of this qualitative approach are subject to my interpretations of the dataset.

Two independent coders (myself and a research assistant) engaged in the initial coding of the transcripts. The research assistant only participated in the initial coding phase for the present study. Two independent coders engaged in initial coding to enhance the trustworthiness of the findings. Initial coding is the process of selecting data related to the study focus and assigning provisional, comparative, and descriptive codes grounded in the data. ${ }^{25} \mathrm{I}$ used line-by-line coding during this process. Line-by-line coding is the process of identifying and describing incidents in the data and then comparing the descriptions of the coded incidents to new datums. ${ }^{26}$ Short, descriptive codes were assigned to each line of data analyzed. Examples of line-by-line codes used include the line "[the physician] went over everything step by step" was coded as "in-depth explanation from physician" and the line "[the physician] was explaining to me what the difference was and why, what the clinical studies were, and why they pushed this to be done" was coded by as "medical explanation for treatment."

I then engaged in Charmaz's $\mathrm{s}^{25}$ process of focused cod- 
ing. Focused coding is the process of categorizing the most significant and repetitive initial codes ${ }^{25}$ I analyzed sets of similar initial codes and then assigned them to a focused code or category. Examples of focused codes used include "in-depth discussions with physicians," "complete patient understanding of the information," "increased time explaining information," and "information presented supportively." Finally, I engaged in theoretical sampling to determine the properties of each category identified in the focused coding process and the connections among them. ${ }^{25} \mathrm{Charmaz}^{25}$ defines theoretical sampling as the process of refining and elaborating on the categories identified to construct the key findings of the analysis. I identified two key themes related to the nature of informational support provided by healthcare providers through the theoretical sampling process. Identification and examination of these themes and their intersections are in the study findings section.

\section{Results}

The research question guiding this study asks what are the characteristics of healthcare provider information-giving processes that breast cancer patients report as indicating support. The analysis of data reveal two related characteristics of provider information-giving processes that participants report as supportive: i) extensive time spent providing cancer-related information, and ii) careful explanation of complex-cancer related information. I also examine the intersections between these related characteristics of informational support.

\section{Extensive time spent providing cancer-related information}

The first characteristic of participants' interactions with providers that they report as being supportive in nature is healthcare providers spending extensive time discussing cancer-related information. Prior research indicates that the duration of an office visit varies widely among cancer patients and physician reimbursement practices may also affect duration of offices visits. ${ }^{27}$ Although it is unclear whether the time providers spend with patients diagnosed with cancer is associated with better health outcomes, interviewees did say that when their physician or other healthcare provider spent extensive time with them they received an increased amount of cancer-related information, that their providers were acting in supportive ways, and that they were better able to make treatment decisions. Thus, a clear commonality among participants' accounts of labeling healthcare providers' information-giving processes as supportive (in contrast to non-supportive) relates to the extensive amount of time those providers spent discussing cancer-related information with the participants.

Participants repeatedly reported that providers' exten- sive time spent delivering information signifies supportive communication. For example, Dolores (76, Stage 1 Breast) said:

I really like nurse practitioners [because they] really have more time and give you a lot more information. I know they are listening to me and understanding what I'm saying too because sometimes you can talk but you can tell [healthcare providers are] not understanding.

Dolores also said that her doctor was "wonderful" because "he will sit and talk to me and ask me if I have any problems too." Dolores' account emphasizes that when healthcare providers are not rushed she can completely share her concerns, ensure that her concerns are understood, and receive complete informational responses.

Other participants similarly noted that when providers spend extensive time providing information they feel cared for and comfortable with their treatment. Julia (50, Stage 2 Breast) explained that her cancer-care team "spends as much time as I need them to be there." Likewise, Elaine (58, Stage 3 Breast) reported that her physician answered all of her questions and "spent that time with me." Ramona (56, Stage 1 Breast) said that the "length of time" her surgeon spent with her going over cancer-related information was "shocking" because she was accustomed to her other physicians (e.g., general practitioners, gynecologists) rushing through the appointment and not receiving enough information.

One exception to this pattern is Lily (49, Stage 1 Breast) who said that "at first" her oncologist was "pretty good" when "explaining" her cancer treatment plan. However, later when she went to her appointments the doctor seemed "rushed" and she "did not always get all the information" she needed or wanted. Lily noticed that she was more nervous about her condition after these short interactions and did not feel that her physician spent the time needed to "explain this to me in a way that I could understand." Lily wanted her providers to "sit down and chat with me for an hour," but felt that either the providers were unable to (because of the number of patients they see) or did not want to spend that kind of time with her. When her providers rushed, Lily saw them as less committed to her as a patient making her feel like she was "a cog" in the "corporate medical giant machine." Thus, when healthcare providers do not spend sufficient time imparting complex cancer-related information, patients accounted for the interaction as lacking support, and a mere function of the provider's job.

Interviewee accounts showed an understanding that providers have significant time constraints and, thus, found the extra time spent with them be unexpected and indicative of support rather than simply as professionals doing their jobs well. Sloan and Knowles' ${ }^{8}$ findings similarly indicate cancer patients perceive healthcare professionals as more caring when they spend time at length (or repeatedly) providing information to them. In addition, 
Sloan and Knowles ${ }^{8}$ also found that when healthcare providers rush through information with patients, patients will feel less satisfied with their interactions and likely not supported.

\section{Careful explanation of complex cancer-related information}

The second characteristic of participants' interactions with providers that they report as being supportive in nature is healthcare providers explaining information carefully in order to ensure that patients understand complex cancer-related information (e.g., diagnosis, side effects, treatment plans). Participants believe that their providers carefully explain cancer-related information because providers want to ensure that they (as patients) are able to understand complex information related to cancer. For example, Carrie (34, Stage 4 Breast) said that her cancer care team was very "caring" and "would explain things and continue on until I understood." Tara (58, Stage 1 Breast) reported that her oncologist "comes in and she's talked to me through everything." Tara continued on to say that because of her oncologist's careful explanations she "felt better going into [treatment]." Stephanie (67, Stage 3 Breast) said, "When they were giving me chemo...[the physician provided a] very good justification, medically...to give me that drug." As such, this explanation helped Stephanie to better understand the reasoning for her treatment.

Nora (72, Stage 1 Breast) said that her doctor was "very good about explaining the radiation, the machines and all of that" so that she "understood" her treatment fully. Nora also noted that she "threw a fit" when tests showed that her "margins were not clear" after her first surgery, but that her doctor showed "great concern" when explaining why she needed additional treatments and it helped Nora to "put things in perspective" and move forward with the treatment. Sandra (55, Stage 1 Breast) reported:

[My oncologist] went out of the way to make sure

I understood what she was talking about... She was ex-

plaining to me what the difference was and why, what

the clinical studies were, and why they pushed this to be done.

Sandra further explained that she was "grateful" to her physician for carefully explaining the rationale for her treatment "because I had a lot of concerns" and "wanted to know the benefits" before making a decision on the type of treatment to choose. She felt that her physician went "above and beyond" her job responsibilities and, as a result, Sandra felt very supported by her physician. Similarly, Lindsay (56, Stage 3 Breast) reported that her surgeon was caring because of the depth with which he presented the information to her. She said, "My surgeon...brought me in the office and he had a booklet [on cancer] there. He went over everything step by step. Explained everything about it. What my type [of cancer] was and all this stuff."
Other participants similarly reported that their healthcare providers were supportive, in contrast to delivering facts about cancer as simply part of their work in instances where providers made sure that patients had fully understood what they said. For example, Tamra (50, Stage 4 Breast) attributed her healthcare providers' actions as motivated by a desire for her to understand the information presented when she said they "make sure I'm understanding everything like my medicines, or if I need anything explained to me or if I need help with getting something medical-wise or anything, they help me with that." Tamra characterized them as "such a supportive team" because they consistently ensured that they met her healthcare needs (informational and otherwise). Ginny's (62, Stage 1 Breast) experience of her oncologist explaining in-depth cancer information thoroughly is particularly unique. Ginny experienced reduced sexual desire as a side effect of her cancer treatment, which caused frustration for her and her partner. Ginny reported strong support from her oncologist when they met to discuss this side effect. She said, "[The physician] would have him come in [to the office] because she wanted him to understand what was going to be happening to me."

An exception to providers thoroughly explaining complex cancer-related information to meet participants' needs included Julia's first conversation with her surgeon. Julia (50, Stage 2 Breast) reported that her surgeon called her while she was at work and told her that she had "cancerous tissue [that was] probably nothing." Julia said that the information was "overwhelming" and that she did not believe that the surgeon had fully explained what her medical situation was and what her options were. In addition, Julia felt the communication of cancer-related information over the phone in contrast to being in person further diminished her ability to fully understand her medical situation. Julia's experience highlights the need for differentiating between information-giving and offering information as supportive communication. That is, the manner providers communicate information may not in fact be supportive, thus increasing uncertainty.

Participants attested that when providers thoroughly explained complex cancer-related information they felt more comfortable making difficult decisions related to their treatment and felt that their providers showed concern for their wellbeing. This second characteristic of supportive information-giving processes relates to the first characteristic (i.e., spending extensive time) identified. Specifically, participants' reports suggest that they are acutely aware of the complicated nature of cancer diagnoses and treatment and have a need for their providers to fully address their concerns (through spending the appropriate amount of time and thoroughly explaining the information) in order to feel comfortable. As such, when providers helped participants to feel at ease with their treatment plans, they more effectively managed the uncertainty related to their diagnosis. 


\section{Metacommunication characterizing provider informational support}

Participants characterized some of the informationgiving dynamics they experienced with healthcare providers (e.g., nurses, physicians) as not only meeting their needs, but as doing so in a manner that also communicated concern and, thereby, went above and beyond what they expected or associated with the healthcare providers' formal responsibilities and were interpreted as supportive. Participants consistently point to the manner (e.g., spending extensive time, explaining information completely, demonstrating concern for patients' informational needs) in which their healthcare providers carried out information-giving when characterizing the messages as being supportive. The intersections between these two characteristics of provider information-giving processes stem from the framing of the content of the message instead of from the explicit content itself (i.e., the information related to the cancer diagnosis or treatment). Thus, it is the metacommunication, or manner in which providers present messages, rather than the content of the messages (i.e., the informational content), that participants interpret as support instead of simply fulfilling job-related information-giving responsibilities.

Wilmot defines metacommunication as anything that 'contextualizes' or 'frames' messages to assist the participants in understanding the communication event. ${ }^{28}$ One could easily argue that providing information in medicine is simply doing one's job and nothing more. However, the study findings suggest that participants draw nuanced distinctions regarding what they expect as part of a provider's information-giving responsibilities in contrast to the presentation of informational messages that demonstrate support for the patient. Notably, participants indicate that not all provider information-giving processes are supportive in nature. Specifically, participant accounts show that providers who rush through information or provide information without sufficient detail are not supportive. As such, these findings suggest that it is the presentation of cancer-related information, or the metacommunication, that informs whether messages are supportive or non-supportive.

\section{Discussion}

Although providing information is arguably a job responsibility for healthcare providers (i.e., providers routinely give health-related information to patients), while providing social support is not, patients in the present exploratory study, and in other studies examining social support and provider-patient communication, ${ }^{4-6,15}$ often characterize providers' information-giving as supportive. However, unlike much of the literature on the informational support provided by healthcare practitioners, the present study differentiates between instances of informa- tional support and information-giving. That is, participants in the present study only classified information as supportive when the provider framed the information in a caring manner.

Participants' accounts suggest that much of the time healthcare providers frame informational messages in ways that indicate that they care about meeting patients' informational needs. For example, participants interpret providers spending extensive time discussing complex cancer-related information with them (instead of feeling rushed) as implicitly conveying care and consideration of their concerns. Thus, patients report that the pace at which the healthcare providers engage in information-giving conveys support. Participants also interpret healthcare providers going out of their way (instead of using medical jargon) to explain cancer-related information in a way that is clear and understandable as demonstrating that the provider cares about their wellbeing (e.g., explaining why a particular medication is used, offering ways to manage painful side effects). The present study emphasizes that metacommunication plays a particularly important role in patients' ability to manage uncertainty related to their diagnosis and treatment. In these specific ways, providers implicitly convey informational content in ways patients deem supportive. Robinson and $\operatorname{Tian}^{6}$ assert that the nature of the relationship between provider and recipient provides the context in which the socially supportive communication occurs and, to some degree, the way the social support will be perceived by the recipient. Thus, when providers interact with patients in ways that communicate concern for the patients' informational needs, patients may believe that they have a more personalized and supportive relationship with the provider instead of a merely formal relationship.

\section{Conclusions}

\section{Study limitations and further research}

The present study includes limitations to consider when interpreting the findings. First, this study was exploratory in nature and, thus, it included a small number of homogenous participants located in one city in the United States. Future studies should include a larger sample size with varied cancer diagnoses, genders, ethnicities, and socioeconomic statuses gathered from a more comprehensive geographical scope. In addition, future research should more closely examine patients' expectations of provider information-giving processes. The interview protocol used in the present study did not explicitly ask participants about their expectations of providers related to information-giving (e.g., do patients expect providers to rush through appointments?). As such, in the future researchers should ask patients diagnosed with cancer whether they expect their providers to interact with them in more supportive ways (especially when providing in- 
formation) because of the serious nature of a cancer diagnosis when compared to the way they interact with providers for other medical reasons (e.g., gynecological needs, yearly check-ups). Finally, future research should examine provider perspectives related to supportive communication and information-giving processes (e.g., do they believe information needs to be given in a supportive manner). Provider perceptions of information-giving would be useful in understanding the challenges and opportunities that healthcare professionals face when meeting patient information needs and performing their job-related tasks.

\section{Practical implications}

Today there are many sources (e.g., healthcare providers, online support groups) of informational support for people diagnosed with breast cancer and, as such, their access to health information has increased substantially. ${ }^{21}$ However, patients often regard healthcare providers as prime source of cancer-related information, such as prognosis and treatment options. ${ }^{3}$ Further, researchers have found that when patients perceive that their providers deliver support they are more likely to express satisfaction with their encounter and even follow directives more closely which may result in better health outcomes. ${ }^{11}$ Thus, the present study findings have important implications for practitioners in two ways. First, they indicate that practitioners can enact specific behaviors (i.e., spending extensive time and thoroughly explaining information) to ensure that they frame informational messages in a supportive manner. As such, practitioners should consider incorporating these communicative strategies when imparting information to breast cancer patients. In particular, communicating cancer-related information in a supportive manner may be beneficial to healthcare providers who are digitally interacting with patients and may have difficulty establishing immediacy the same way they would be able to in face-to-face encounters. Second, practitioners who practice supportive information-giving behaviors may find that their patients are more comfortable and receptive to the information provided and may be more likely to follow provider guidance (e.g., treatment plans, lifestyle changes). Thus, providers should not discount the importance of acting in supportive ways when communicating cancer-related information and they should work to enact these behaviors to ensure that they meet patients' informational needs.

\section{References}

1. Department of Health and Human Services, Centers for Disease Control and Prevention, and National Cancer Institute. U.S. cancer statistics: 1999-2014. Incidence and mortality web-based report 2017.

2. Wolff AC, Domchek SM, Davidson NE, et al. Cancer of the breast. In: Niederhuber JE, Armitage J, Doroshow J, et al., eds. Abeloff's Clinical Oncology. 5th ed. Philadelphia, Pa: Churchill Livingstone Elsevier; 2014. pp 1630-1752.

3. Rutten LJF, Arora NJ, Bakos AD, et al. Information needs and sources of information among cancer patients: A systematic review of research (1980-2003). Patient Educ Couns 2005;57:250-61.

4. Dakof GA, Taylor SE. Victims' perceptions of social support: What is helpful from whom? J Pers Soc Psychol 1990; $1: 80-9$

5. Nazione S, Silk KJ, Robinson J. Verbal social support for newly diagnosed breast cancer patients during surgical decision-making visits. J Commun Health 2016;9:267-78.

6. Robinson JD, Tian Y. Cancer patients and the provision of informational social support. Health Commun 2009;24:381-90.

7. Robinson JD, Turner J. Impersonal, interpersonal, and hyperpersonal social support: Cancer and older adults. Health Commun 2003; 15:227-34.

8. Sloan AG, Knowles A. Improving communication between healthcare providers and cancer patients: A pilot study. J Commun Health 2013;6:208-15.

9. Dunkel-Schetter C. Social support and cancer: Findings based on patient interviews and their implications. J Soc Issues 1984;40:77-98.

10. Sanson-Fisher R, Girgis A, Boyes A, et al. The unmet supportive care needs of patients with cancer. Cancer 2000;88:225-36.

11. Mills ME, Sullivan K. The importance of information giving for patients newly diagnosed with cancer: A review of literature. J Clin Nurs 1999;8:631-42.

12. Albrecht TL, Goldsmith DJ. Social support, social networks, and health. In: Thompson TL, Dorsey A, Miller KI, Parrott R, eds. Handbook of health communication. Mahwah, NJ: Lawrence Erlbaum; 2003. pp 263-284.

13. Albrecht TL, Adelman MB. Communicating social support: A theoretical perspective. In: Albrecht TL, Adelman MB, eds. Communicating social support. Newbury Park, CA: Sage; 1987. pp 18-39.

14. Brashers DE. Communication and uncertainty management. J Commun 2001;51:477-97.

15. Palmer-Wackerly AL, Krieger JL, Rhodes ND. The role of health care provider and partner decisional support in patients' cancer treatment decision-making satisfaction. J Health Commun 2017;22:10-9.

16. Innes S, Payne S. Advanced cancer patients' prognostic information preferences: A review. Palliat Med 2009;23:29-39.

17. Cobb S. Social support as a moderator of life stress. Psychosom Med 1976;38:300-14.

18. Barrera M, Ainlay SL. The structure of social support: A conceptual and empirical analysis. J Community Psychol 1983;11:133-43.

19. Cutrona CE, Suhr JA. Controllability of stressful events and satisfaction with spouse support behaviors. Commun Res 1992;2:154-74.

20. Goldsmith DJ. Communicating social support. New York, NY: Cambridge University Press; 2004.

21. Wright KB, Johnson AJ, Bernard DR, Averbeck J. Computer-mediated social support: Promises and pitfalls for individuals coping with health concerns. In: Thompson TL, Parrott R, Nussbaum JF, eds. The Routledge handbook of health communication. 2nd ed. New York, NY: Routledge; 2011. pp 349-362.

22. McPherson CJ, Higginson IJ, Hearn J. Effective methods of 
giving information in cancer: A systematic literature review of randomized controlled trials. J Pub Health Med 2001;23:227-34.

23. Jenkins K, Fallowfield L, Saul J. Information needs of patients with cancer: Results from a large study in UK cancer centres. Br J Cancer 2001;84:48-51.

24. Johnson JM, Rowlands, T. The interpersonal dynamics of in-depth interviewing. In: Gubrium JF, Holstein JA, Marvasti $\mathrm{AB}$, McKinney $\mathrm{KD}$, eds. The SAGE handbook of interview research: The complexity of the craft. Thousand Oaks, CA: SAGE Publications Inc.; 2012. pp 99-114.
25. Charmaz K. Constructing grounded theory. 2nd ed. Thousand Oaks, CA: SAGE; 2014.

26. Charmaz K. Constructing grounded theory: A practical guide through qualitative analysis. Thousand Oaks, CA: SAGE; 2006.

27. Guy GP, Richardson, LC. Visit duration for outpatient physician office visits among patients with cancer. J Oncol Pract 2012;8:2s-8.

28. Wilmot WW. Metacommunication: A re-examination and extension. In: Nimmo D, ed. Communication Yearbook 4. New Brunswick, NJ: Transaction Books; 1980. pp 61-69. 